Pacific Journal of Mathematics

ARC-WISE CONNECTEDNESS IN SEMI-METRIC SPACES 


\title{
ARC-WISE CONNECTEDNESS IN SEMI-METRIC SPACES
}

\author{
ROBERT W. HEATH
}

1. Introduction. The Arc Theorem usually encountered is the following: a connected and locally connected Cauchy complete metric ${ }^{1}$ space is arc-wise connected [10]. The most general Arc Theorem is Theorem 1 in Chapter II of [14], in which "Cauchy complete metric space" is replaced by "a space satisfying Moore's Axiom 1"-i.e. a "complete Moore space" (equivalent to a complete regular developable space [1]; see also [16] and [15]). Wyman Richardson, in one of F. B. Jones' classes proved the Arc Theorem for strongly complete regular semimetric spaces (unpublished though the argument differed considerably from Moore's argument). This was not, however, a real generalization because such spaces are Moore spaces (cf. Corollary 4.3 of this paper).

Since most theorems which are true in Moore spaces are true in regular semi-metric ${ }^{1}$ spaces, and since the exceptions are "in general those theorems whose validity depends upon that property of Moore spaces which forces the equivalence of perfect and hereditary separability" [7], one might hope that the Arc Theorem could be further generalized by simply replacing "metric" by "regular semi-metric." This paper establishes that the Arc Theorem cannot be generalized directly to Cauchy complete regular semi-metric spaces but can be extended to a somewhat more general class of regular semi-metric spaces then those satisfying Moore's Axiom 1. The examples given show that, even in the presence of such properties as possessing a uniformity and being compactly connected, a regular semi-metric space can be Cauchy complete, connected and locally connected but not be arc-wise connected. Other possible means of extending the Arc Theorem are eliminated by establishing that in the presence of certain topological properties a regular semimetric space is a Moore space (e.g. a strongly complete semi-metric space is a Moore space)-or is even metrizable.

This paper is essentially a dissertation [4] written at the University of North Carolina under the direction of Professor F. B. Jones. The

Received July 22, 1960, and in revised form May 17, 1961, and December 20, 1961. Presented to the American Mathematical Society, June 18, 1960.

1 A topological space $S$ is said to be semi-metric if there is a distance function $d$ for $S$ with respect to which the topology of $S$ is invariant. A distance function $d$ for $S$ is a function from $S \times S$ to the nonnegative numbers such that, if each of $x$ and $y$ is a point of $S$, then $(1) d(x, y)=0$ only in case $x=y$ and $(2) d(x, y)=d(y, x)[11 ; 18]$. The space is metric if the distance function also satisfies $(3) d(x, y)+d(y, x) \geqq d(x, z)$ for each triple $x, y, z$ of points of $S$. Note that every Moore space is regular and semi-metric. The set $U_{c}(x)=\{y: d(x, y)<c\}$ is referred to herein as a $c$-neighborhood (with respect to $d$ ) of $x$. Cauchy complete is defined as in [11, p. 316]. Topological space and regular are defined as in [9, pp. 37 and 113]. Terms not defined herein are used as in [14], [11], or [1]. 
author wishes to thank Professor Jones for his encouragement and direction.

2. Cauchy complete semi-metric spaces in which the Arc Theorem does not hold true. The following examples and theorems show that a Cauchy complete regular semi-metric space may be connected and locally connected (and even compactly connected) without being arc-wise connected. Example 2.2 is such a space, some additional properties of which are given in Theorem 2.3. Example 2.5 is such a space which is compactly connected. In the remainder of this section some additional properties of those spaces are pointed out to show that those properties could not be used to extend the Arc Theorem, and there are described some other spaces which are practically indistinguishable from the first two spaces but which are arc-wise connected. The following definition will be useful since weak completeness is equivalent to Cauchy completeness [11, Theorem 2.3].

Definition 2.1. A space $S$ is said to be $\left\{\begin{array}{l}\text { weakly complete } \\ \text { strongly complete }\end{array}\right\}$ provided there exists a distance function $d$ such that (1) the topology of $S$ is invarient with respect to $d$ and (2) if $M$ is a nonincreasing sequence of closed sets in $S$ such that, for each $n$, there is a $1 / n$-neighborhood of a point $p_{n}\left\{\begin{array}{ll}\text { in } & M_{n} \\ \text { in } & S\end{array}\right\}$ which contains $M_{n}$, then $\prod_{n=1}^{\infty} M_{n}$ contains a point.

EXAMPLE 2.2. Let $S$ consist of the points of $[0,1] \times[0,1]$ with a distance function $d$ and a topology defined as follows.

(1) If $x \in S, d(x, x)=0$, and

(2) if $x$ and $y$ are two points of $S$ and $a(x, y)$ is the smallest nonnegative angle (in radians) formed by the line which contains $x$ and is parallel to $X$ (the $x$-axis), or is $X$, and the line which contains $x$ and $y$, then $d(x, y)=|x-y|+a(x, y)$. For each point $p$ of $S$ and each positive number $c$, let the $c$-neighborhood of $p, U_{c}(p)=\{x: d(x, p)<c\}$, be an element of a basis for the topology of $S$.

Clearly the semi-metric space $S$ is weakly complete (hence Cauchy complete), completely regular (hence uniform and, of course, regular; cf. [9]) and separable. That $S$ is connected and locally connected follows from the fact that horizontal line segments in $[0,1] \times[0,1]$ have the same relative topology in $S$ as in Euclidean two-space.

Note that, if $(b, c)$ is a point of $S$, if $d>0$, if $0<a<\pi / 2$, and if $R(b, c ; a, d)=[\{(x, y):|x-b|<d$, and either $|y-c|<|x-b| \tan a$ or $y=c\}] \cdot S$-i.e. if $R(b, c ; a, d)$ is the point set consisting of $(b, c)$ and all points of $S$ interior to a (horizontally oriented) "bow-tie region" centered on $(b, c)$ and having (horizontal) length $d$ and central angles 
of magnitude $2 a$ radians - then $R(b, c ; a, d)$ is an open set in $S$. Furthermore, the collection $\{R(b, c ; a, d):(b, c) \in S, d>0$ and $0<a<\pi / 2\}$ of all such bow-tie regions in $S$ forms a basis for the topology of $S$. That basis is useful in the proof of Theorem 2.3.

The proof of the following lemma, as well as a more detailed proof of Theorem 2.3, is contained in the proof of Theorem 1 [4, p. 10].

Lemma. If $M$ is an arc in $S$ with nondegenerate $x$ and $y$ projections, then there is a subarc $M_{1}$ of $M$ with nondegenerate $x$ and $y$ projections and whose $x$-projection is a subset of the $x$-projection of $M-M_{1}$.

THEOREM 2.3. There exists a separable, connected, locally connected, weakly complete, completely regular, semi-metric space which is not arc-wise connected.

Proof. Let $S$ be the space of Example 2.2. The space $S$ is not arc-wise connected. For suppose that there is an arc in $S$ with endpoints $(0,0)$ and $(1,1)$.

By the above lemma, if $M$ is an arc in $S$ and with endpoints $(0,0)$ and $(1,1)$, there is a sequence $\left\{M_{i}\right\}_{i=1}^{\infty}$ of subarcs of $M$ such that, for each $n, M_{n} \supset M_{n+1}$ and $\left\{x:(x, y) \in M_{n+1}\right\} \subset\left\{x:(x, y) \in\left[M_{n}-M_{n+1}\right]\right\}$. Then, since $M$ is compact, there is a point $(p, q)$ in $\prod_{i=1}^{\infty} M_{i}$, and, for each $n$, there is a point $\left(p, q_{n}\right)$ in $M_{n}$ such that $q_{n}=q_{m}$ only if $n=m$. Thus $M$ contains an infinite subset without a limit point which violates the compactness of $M$.

DeFinition 2.4. A space $S$ is said to be compactly connected provided that, if $a$ and $b$ are points of $S, S$ contains a compact continuum which contains both $a$ and $b$.

There are now two questions to be answered. Is a connected, locally connected, weakly complete, regular (or completely regular) semimetric space compactly connected? Also, is a connected, locally connected, weakly complete, regular (or completely regular) semi-metric space which is compactly connected necessarily arc-wise connected? The answer to both questions is no. It can be shown that the space of Example 2.2 is not compactly connected by an argument in general following the same outline as the proof of Theorem 2.3-replacing subarc by irreducible subcontinuum and making use of Theorems 32, 39, and 47 from Chapter I of Moore's Foundations (for a detailed proof see Theorem 2 [4, p. 13]). Example 2.5 and Theorem 2.6 answer the second question.

EXAMPLe 2.5. Let $K$ be the "polyhedral $\sin 1 / x$ curve" in $[0,1] \times$ 
$[0,1]$ which is the union of all horizontal line segment of the form

$$
\{x: 0 \leqq \operatorname{Re}[x] \leqq 1 \text { and } \operatorname{Im}[x]=0\}
$$

or

$$
\begin{aligned}
& \{x: 0 \leqq \operatorname{Re}[x] \leqq 1 \text { and } \operatorname{Im}[x]=1 / 2 n\} \\
& \text { for } n=0,1,2, \cdots,
\end{aligned}
$$

and of all vertical line segments of the form

$$
\left\{x: \operatorname{Re}[x]=0, \frac{1}{2^{2 m+1}} \leqq \operatorname{Im}[x] \leqq \frac{1}{2^{2 m}}\right\} \quad \text { for } \quad m=0,1,2, \cdots
$$

or

$$
\left\{x: \operatorname{Re}[x]=1, \frac{1}{2^{2 m}} \leqq \operatorname{Im}[x] \leqq \frac{1}{2^{2 m-1}}\right\} \quad \text { for } \quad m=1,2,3, \cdots
$$

Let $d$ be the distance function for $[-2,2] \times[0,1]$ defined as follows:

(1) if $x$ is a point of $K$ and $y$ is a point of $[-2,2] \times[0,1]$, then $d(x, y)=|x-y|$

(2) if $x$ and $y$ are points of $([-2,2] \times[0,1]-K)$ and $a(x, y)$ is the smallest nonnegative angle (in radians) formed by the line $x y$ and the horizontal, then $d(x, y)=|x-y|+a(x, y)$.

Let $S$ be the topological space consisting of the set $[-2,2] \times[0,1]$ with the following topology: for each point $p$ of $K$ and each $c>0$, the circular neighborhood $\{x: x \in S,|x-y|<c\}$ is a region in $S$, and, if $p$ is a point of $S$ but not of $K$, every "bow-tie region" (as defined in Example 2.2) with center at $p$ is a region in $S$. Clearly $S$ is a completely regular semi-metric space which is weakly complete, connected, locally connected, and separable.

THEOREM 2.6. There is a connected, locally connected, weakly complete, completely regular semi-metric space which is compactly connected but not arc-wise connected.

Proof. Let $S$ be the space defined in Example 2.5. Since $K$ has the same relative topology as it would in the usual plane topology, $K$ is a compact continuum; likewise each horizontal interval contained in $S$ is a compact continuum. Hence, if $a$ and $b$ are points of $S$, the point set $[K+\{x: x \in S$ and $\operatorname{Im}[x]=\operatorname{Im}[a]\}+\{x: x \in S$ and $\operatorname{Im}[x]=\operatorname{Im}[b]\}]$ is a compact continuum which contains $a$ and $b$ and is contained in $S$. Thus $S$ is compactly connected.

From the proof of Theorem 2.3, it is clear that any nondegenerate 
compact continuum in $S$, other than a horizontal interval, must intersect $K$. Therefore, a compact continuum $L$ in $S$ which contains the points $(2,1)$ and $(-2,0)$ must contain $K$; but each point of $\{z: z \in K, 0<\operatorname{Re}[z]<1$ and $\operatorname{Im}[z]=0\}$ is a nonseparating point of $K$ and is not a boundary point of any component of $L-K$ (and therefore a nonseparating point of $L)$. Hence there is no arc in $S$ which contains $(2,1)$ and $(-2,0)$.

Returning to the space $S$ of Example 2.2, it is perhaps of interest whether points of that space are connectable by any type of sets bearing some resemblance to arcs. It will be shown in $\S \mathrm{VI}$ that, if $a$ and $b$ are points of $S$, there is a continuum $M$ in $S$ whose only nonseparating points are $a$ and $b$. It can also be shown that, if $a$ and $b$ are points of $S$, then either $a$ and $b$ are endpoints of an arc in $S$ (in case $a$ and $b$ are on the same horizontal line segment), or $a$ and $b$ are the only nonseparating points of a connected subset $M$ of $S$ which is the graph of a function, namely $M^{-1}=\{(x, y):(y, x) \in M\}$ is a function. The existence of the latter can be established by an argument somewhat similar to the proof of Theorem 5 in [6]. For a detailed proof see [4, pp. 20-24].

Consider now the following two examples each of which is a connected, locally connected, weakly complete, completely regular, semimetric space which closely resembles Example 2.2, and each of which also is neither a Moore space nor strongly complete (nor complete in any of the "intermediate" senses to be subsequently defined), but each of which is arc-wise connected.

Example 2.7. Define a distance function $d$ for the points of $[0,1] \times$ $[0,1]$ as follows: if $x$ and $y$ are two points of $[0,1] \times[0,1]$, then (1) if $\operatorname{Re}[x]=\operatorname{Re}[y], d(x, y)=1$, and (2) if $\operatorname{Re}[x] \neq \operatorname{Re}[y]$, then $d(x, y)=$ 2(g.l.b. $[c: c>0$ and $y \in\{z:|z-(x+c)|<c$ or $|z-(x-c)|<c\}])=$ $|x-y|^{2} /|\operatorname{Re} x-\operatorname{Re} y|=|x-y| \sec a$, where $a$ is the smallest nonnegative angle formed by the line passing through $x$ and $y$ and the horizontal line through $x$. Note that neighborhoods of radius less than 1 are "bow-ties" formed by tangent circles (the center of such a neighborhood is the point of tangency, and, if the neighborhood has center $x$ and radius $2 c$, the centers of the circles are $x-c$ and $x+c$ and the radius of each circle is $c$ ). The topological space $S$ consisting of the points of $[0,1] \times[0,1]$ and regions which are (all) such neighborhoods is a connected, locally connected, weakly complete, completely regular semi-metric space by the same arguments as used in Example 2.2. That $S$ is neither strongly complete nor a Moore space will be more easily seen following subsequent theorems. The space $S$ is arc-wise connected since every nonvertical line segment in $[0,1] \times[0,1]$ has the same relative topology in $S$ as it does in the plane topology, and hence is an are in $S$.

The second example is due to L. F. McAuley [11]. 
Example 2.8. Let $X$ denote the $x$-axis of the Cartesian plane $E^{2}$. Define a distance function $d$ for the points of $E^{2}$ as follows: if each of $p$ and $q$ is a point of $E^{2}$, then

(1) if neither $p$ nor $q$ belongs to $X$ or if both belong to $X, d(p, q)=$ $|p-q|$, and

(2) if exactly one of $p$ any $q$ belongs to $X, d(p, q)=|p-q|+a$ where $a$ is the nonobtuse angle (measured in radians) between $X$ and the line $L$ determined by $p$ and $q$. Thus a neighborhood of a point of $X$ is a "bow-tie" neighborhood while a neighborhood of a point not on $X$ is a disc (with some distortion in case the neighborhood intersects $X)$. The topological space $S$ consisting of the points of $E^{2}$ and regions which are $d$-neighborhoods is clearly a connected, locally connected, weakly complete, completely regular semi-metric space that is arc-wise connected.

Each of the distance functions defined in Examples 2.2, 2.7, and 2.8 has the following continuity property: (in each case $d$ denotes the distance function for the space $S$ ) if $x$ and $y$ are point sequences in $S$ which have respective sequential limit points $p$ and $q$ such that $p \neq q$, then $\lim _{n \rightarrow \infty} d\left(x_{n}, y_{n}\right)=d(p, q)$. It is easily shown that, if $S$ is a regular semi-metric space with a distance function which has the above continuity property, then neighborhoods with respect to that function are open sets and the closure of a compact set in $S$ is compact.

Each of the distance functions defined in Examples 2.7 and 2.8 has in addition the following "convexity" property. If $a$ and $b$ are two points of $S$ such that $d(a, b)<1$ and $n$ is a natural number, then there is a point sequence $a_{0}, a_{1}, a_{2}, \cdots, a_{n}$ in $S$ such that $a_{0}=a, a_{n}=b$ and, if $0 \leqq i<j<k \leqq n, d\left(a_{i}, a_{j}\right)+d\left(a_{j}, a_{k}\right)=d\left(a_{i}, a_{k}\right)$, and $a_{i+1}$ is the only point of $S$ such that $d\left(a_{i}, a_{i+1}\right)=d\left(a_{i+1}, a_{i+2}\right)=1 / 2 d\left(a_{i}, a_{i+2}\right)$. That property, plus the properties that neighborhoods are connected open sets and that the closure of a compact set is compact, is a sufficient condition for the (weakly complete, regular semimetric) spaces of Examples 2.7 and 2.8 to be arc-wise connected.

3. Conditions for semi-metric, developable, and metric spaces. Among the open questions about semi-metric spaces are the following. Is there a "purely topological" characterization of semi-metric spaces [12], and what "topological" property can be added to a semi-metric space to get a developable [1, p. 180] (or Moore) space [2, p. 64]? The answers to those questions, or at least some uniform characterization, of semi-metric, developable and metric spaces, should be useful in trying to extend Moore's Arc Theorem. The author found the characterization given below by the Conditions A, B and C useful not only for that purpose, but also for easy construction of nondevelopable semimetric spaces as well as nonmetric Moore spaces (in trying to generalize 
the Arc Theorem by weakening the completeness part of Moore's Axiom 1). For another application see [5].

A second set of topological conditions, $\mathrm{A}^{\prime}$ and $\mathrm{B}^{\prime}$, is obtained by weakening Conditions $\mathrm{A}$ and $\mathrm{B}$. Condition $\mathrm{A}^{\prime}$ is the topological axiom used in the Arc Theorem given in $\S \mathrm{VI}$; and Theorem 3.5 establishes that a regular $T_{1}$-space satisfying Conditions $\mathrm{A}$ and $\mathrm{B}^{\prime}$ is a Moore spacefrom which it follows (in $\S I V$ ) that a strongly complete regular semimetric space is a Moore space (Theorem 2.2 in [11] is a corollary to this theorem). Theorem 3.6 establishes that every semi-metric space has a property analogous to that characterizing property of metric spaces pointed out in [8] and to the similar characterizing property of Moore spaces implicit in Moore's Axiom 1.

Throughout this section $Z$ denotes the set of all natural numbers, and a $T_{1}$-space (also $T_{2}, T_{3}$, etc.) is as defined in [9, p. 56]. The following definition is also used.

DEFINITION 3.1. A sequence $x$ of points in the space $S$ converges to a point $y$ of $S$ only in case every region which contains $y$ contains $x_{i}$ for all but finitely many values of $i ; y$ is then called a sequential limit point of $x$.

Suppose that $S$ is a $T_{1}$-space. Consider the following three conditions on a function $g$ from $Z \times S$ to the collection of all open sets in $S$.

Condition A. (1) For each point $x$ of $S,\left\{g_{n}(x)\right\}_{n=1}^{\infty}$ is a nonincreasing sequence which forms a local base for the topology at $x$. (2) If $y$ is a point of $S$ and $x$ is a point sequence in $S$ such that, for each natural number $m, y \in g_{m}\left(x_{m}\right)$, then $x$ converges to $y$.

Condition B. If $y$ is a point of $S$ and $x$ and $z$ are point sequences in $S$ such that, for each $m,\left[y+x_{m}\right] \subset g_{m}\left(z_{m}\right)$, then $x$ converges to $y$.

Condition C. If each of $x$ and $y$ is a point of $S$ and $n$ is a natural number such that $x \in g_{n}(y)$, then $y \in g_{n}(x)$. [cf. 3, p. 257 and p. 261].

THEOREM 3.2. A necessary and sufficient condition that a $T_{1}$-space $S$ be semi-metric is that there is a function $g$, from $Z \times S$ to the open sets of $S$, such that $g$ satisfies Condition $A$.

Proof. The condition is sufficient. For suppose that there is a function $g$ which satisfies Condition A. Define the function $m$, from $S \times S$ to the natural numbers, as follows: if $x$ and $y$ are two points of $S, m(x, y)$ is the smallest natural number $p$ such that $y \notin g_{p}(x)$. Define 
a distance function $d$ for $S$ as follows: if $x \in S, d(x, x)=0$; if $x$ and $y$ are two points of $S, d(x, y)=\min [1 / m(x, y), 1 / m(y, x)]=$ the reciprocal of the smallest natural number $p$ such that $y \notin g_{p}(x)$ and $x \notin g_{p}(y)$. Clearly, if each of $x$ and $y$ is a point of $S, d(x, y)=d(y, x)$; and $d(x, y)=0$ only if $x=y$. Also limit points are invariant with respect to $d$ (for a proof see $[4$, p. 30]).

The condition is necessary. For suppose that $S$ is a semi-metric space. Define the function $g$ as follows: if $x$ is a point of $S$ and $n$ is a natural number, $g_{n}(x)=$ interior $\left[U_{1 / n}(x)\right]=\{y$ : for some region $R$, $\left.y \in R \subset U_{1 / n}(x)\right\}$. Clearly $g$ satisfies Condition A.

THEOREM 3.3. A necessary and sufficient condition that a $T_{1}$-space $S$ be developable is that there be a function $g$, from $Z \times S$ to the open sets of $S$, such that $g$ satisfied Conditions $A$ and $B$.

Proof. The condition is sufficient. For suppose that there is a function $g$ satisfying Conditions $\mathrm{A}$ and $\mathrm{B}$. For each natural number $i$, let $G_{i}=\left\{g_{j}(x): x \in S, j \geqq i\right\}$. The coverings $G_{1}, G_{2}, G_{3}, \cdots$ constitute a development (for proof see [4, p. 32]).

Suppose, conversely, that $G_{1}, G_{2}, \cdots$ is a development for $S$. Define the function $g$ as follows: for each point $x$ of $S$ let $g_{1}(x)$ be some member of $G_{1}$ which contains $x$, and, if $n$ is a natural number greater than 1 , let $g_{n}(x)$ be a member of $G_{n}$ such that $x \in g_{n}(x) \subset g_{n-1}(x)$. Clearly $g$ satisfies Conditions $\mathrm{A}$ and $\mathrm{B}$.

THEOREM 3.4. A necessary and sufficient condition that a $T_{1}$-space $S$ be metric is that there is a function $g$, from $Z \times S$ to the open sets of $S$ such that $g$ satisfies Conditions $A, B$, and $C$.

Proof. The condition is necessary, for suppose that $S$ is a metric space. Define the function $g$, from $Z \times S$ to the open sets of $S$, as follows: for each point $x$ and natural number $n, g_{n}(x)=$ interior $\left[U_{1 / n}(x)\right]$. It is clear that $g$ satisfies Conditions $\mathrm{A}, \mathrm{B}$, and $\mathrm{C}$.

Conversely, let $S$ be a $T_{1}$-space, and let $g$ be a function which satisfies Conditions A, B, and C. For each $n$, let $G_{n}=\left\{g_{m}(x): x \in S\right.$, $m \geqq n\}$. By Moore's metrization theorem [11, p. 325], if $S$ is not metrizable, there are two points $p$ and $q$ and a region $R$ such that, for each $n, G_{n}$ contains members $h$ and $k$ such that $p \in h, h \cdot k \neq 0$, and $k \cdot[S-(R-q)] \neq 0$ (i.e., $K \cdot(S-R) \neq 0$ since $S$ is $\left.T_{1}\right)$. Thus there are a point $p$, a region $R$, and point sequences $x, y$, and $z$ such that, for each $n, p \in g_{n}\left(x_{n}\right), y_{n} \in g_{n}\left(x_{n}\right) \cdot g_{n}\left(z_{n}\right)$, and $g_{n}\left(z_{n}\right) \cdot[S-R] \neq 0$. By Condition $\mathrm{B},\left[y_{n}+p\right] \subset g_{n}\left(x_{n}\right)(n=1,2,3, \cdots)$ implies that the sequence $y$ converges to $p$. Therefore, there is an increasing natural number sequence $m$ such that, for each natural number $n, y_{m(n)} \in g_{n}(p)$, so, that, 
by Condition C, $p \in g_{n}\left(y_{m(n)}\right)$. Also $y_{m(n)} \in g_{m(n)}\left(Z_{m(n)}\right)$ implies that $z_{m(n)} \in$ $g_{m(n)}\left(y_{m(n)}\right)$-hence that $z_{m(n)} \in g_{n}\left(y_{m(n)}\right)$.

Thus, for each $n,\left[p+z_{m(n)}\right] \subset g_{n}\left(y_{m(n)}\right)$, so that, by Condition $\mathrm{B}$, $\left\{z_{m(n)}\right\}_{n=1}^{\infty}$ converges to $p$. Therefore there is a subsequence $r$ of $m$ such that, for each natural number $n, z_{r(n)} \in g_{n}(p)$, hence $p \in g_{n}\left(z_{r(n)}\right)$. But by supposition there is a point sequence $u$ such that, for each $n, u_{n} \in(S-R)$ and $u_{n} \in g_{n}\left(z_{r(n)}\right)$, so that $u$ converges to $p$; which leads to the contradiction that $p \in \overline{(S-R)}$ while $p$ is contained in the region $R$. Thus $S$ must be metrizable.

Consider Conditions $\mathrm{A}^{\prime}$ and $\mathrm{B}^{\prime}$ which are at least formally weaker than $\mathrm{A}$ and $\mathrm{B}$ respectively.

Condition A'. (1) For each point $x$ of $S,\left\{g_{n}(x)\right\}_{n=1}^{\infty}$ is a nonincreasing sequence which forms a local base for the topology at $x$. (2) If $y$ is a point of $S$ and $x$ is a point sequence such that, for each natural number $n, y \in g_{n}\left(x_{n}\right)$ and there is a natural number $k$ such that $\overline{g_{n+k}\left(x_{n+k}\right)} \subset g_{n}\left(x_{n}\right)$, then the sequence $x$ converges to $y$.

Condition B'. If $y$ is a point of $S, R$ is an open set containing $y$, and $x$ is a point sequence such that, for each $n, y \in g_{n}\left(x_{n}\right)$ and there is a $k$ such that $\overline{g_{n+k}\left(x_{n+k}\right)} \subset g_{n}\left(x_{n}\right)$, then there is a natural number $m$ such that $g_{m}\left(x_{m}\right) \subset R$.

It will be convenient now to have Condition $\mathrm{A}, \mathrm{A}^{\prime}, \mathrm{B}, \mathrm{B}^{\prime}$, and $\mathrm{C}$ translated into corresponding conditions on a basis for the space.

Definition 3.5. Suppose that $S$ is a $T_{1}$-space and that $G$ is a basis for $S$. The basis $G$ satisfies Condition A $\left(\mathrm{A}^{\prime}, \mathrm{B}, \mathrm{B}^{\prime}\right.$, or $\left.\mathrm{C}\right)$ means that there is a function $g$, from $Z \times S$ to the open sets of $S$, such that $G=\left\{g_{n}(x): x \in S, n=1,2,3, \cdots\right\}$ and $g$ satisfies Condition $\mathrm{A}\left(\mathrm{A}^{\prime}, \mathrm{B}, \mathrm{B}^{\prime}\right.$, or $\mathrm{C})$.

Theorem 3.6 establishes that the Arc Theorem cannot be generalized by simply replacing "Moore space" by "a regular semi-metric space satisfying Condition $\mathrm{B}^{\prime \prime}$ " since such a space is itself a Moore space; moreover it will readily follow from this theorem (cf. Corollary 4.3) that every strongly complete regular semi-metric space is a Moore space, thus eliminating another means of extending the Arc Theorem as well as improving upon Theorem 2.2 of [11].

THEOREM 3.6. Suppose that $G$ is a basis for a regular $T_{1}$-space $S$. If $G$ satisfies Condition $A$ and $B^{\prime}$, then $S$ has a basis $H$ which satisfies Conditions $A$ and $B$, hence $S$ is a Moore space.

Proof. Suppose that the regular $T_{1}$-space $S$ has a basis $G=$ 
$\left\{g_{m}(x): x \in S, m=1,2, \cdots\right\}$ which satisfies Conditions $\mathrm{A}$ and $\mathrm{B}^{\prime}$. Let $\alpha=\left\{p_{1}, p_{2}, \cdots\right\}$ be a well-ordering of the points of $S$. Define the functions $h$ (from $Z \times S$ to $G$ ), $r$ (from $\alpha \times Z$ to $Z$ ), and $n$ (from $\alpha x$ : [a subset of $Z$ ] to $\alpha$ ) as follows. For each $p_{z} \in S$, let $h_{1}\left(p_{z}\right)=g_{r_{z}[1]}\left(p_{z}\right)=$ $g_{1}\left(p_{z}\right)$. For each $p_{z} \in S$ and each natural number $i$ greater than 1:

(Case 1) If there is not a point $q$ of [S- $\left.p_{z}\right]$ and a natural number $j<i$ such that $p_{z} \in h_{j}(q)$ and $h_{j}(q) \cdot\left[S-g_{r_{z}[i-1]+1}\left(p_{z}\right) \neq 0\right.$, then let $h_{i}\left(p_{z}\right)=$ $g_{r_{z}[i]}\left(p_{z}\right)=g_{r_{z}[i-1]+1}\left(p_{z}\right)$; or

(Case 2) otherwise, for each (such) $j<i$, let $p_{n_{z}[j]}$ be the first. member $q$ of $\alpha\left(q \neq p_{z}\right)$ such that $p_{z} \in h_{j}(q)$ and $h_{j}(q) \cdot\left[S-g_{r_{z}[i-1]+1}\left(p_{z}\right)\right] \neq 0$; let $r_{z}[i]$ be the smallest natural number $m>r_{z}[i-1]$ such that $\overline{g_{m}\left(p_{z}\right)} \subset$ $\Pi\left[h_{j}\left(p_{n_{z}[j]}: j<i\right.\right.$, and $j$ is not covered by Case 1]; and let $h_{i}\left(p_{z}\right)=$ $g_{r_{z}[i]}\left(p_{z}\right)$.

The basis $H=\left\{h_{i}(x): x \in S, i=1,2, \cdots\right\}$ then satisfies Conditions A and $\mathrm{B}^{\prime}$, since $H$ is a subcollection of $G$ and since, if $x \in g \in G$, then there is an $h \in H$ such that $x \in h \subset g$.

The basis $H$ satisfies Condition B. For if not, then there is a point $x$ of a region $R$ such that, for each $m$, there is a point $q$ such that $x \in h_{m}(q)$ and $h_{m}(q) \cdot[S-R] \neq 0$. Let $y$ be the point sequence such that, for each $m, y_{m}$ is the first point $q$ in $\alpha$ such that $x \in h_{m}(q)$ and $h_{m}(q)$. $[S-R] \neq 0$. (It will now be shown that, for each natural number $i$, there is an $m>i$ such that $\overline{h_{m}\left(y_{m}\right)} \subset h_{i}\left(y_{i}\right)$.)

If $i$ is a natural number there is a natural number $N_{1}>i$ such that, if $m>N_{1}$, then $y_{m} \in\left[h_{i}\left(y_{i}\right)-y_{i}\right]$ (since $y$ converges to $x$ and $\left.x \in h_{i}\left(y_{i}\right)\right)$ and there is an $N_{2}$ such that, if $m>N_{2}$, then $g_{r_{\left(y_{m}\right)}[m-1]+1}\left(y_{m}\right)$ does not contain $h_{i}\left(y_{i}\right)$ (otherwise, by Condition A, each point of $h_{i}\left(y_{i}\right)$ would be a sequential limit point of $y$, and $h_{i}\left(y_{i}\right)$ contains at least two points, namely, $x$ and a point not in $R$ ); thus there is a natural number $m$ such that

$$
y_{m} \in\left[h_{i}\left(y_{i}\right)-y_{i}\right] \text { and } h_{i}\left(y_{i}\right) \cdot\left[S-g_{r_{\left(y_{m}\right)}[m-1]+1}\left(y_{m}\right)\right] \neq 0 \text {. }
$$

Moreover, there is no point $q$ such that $q$ precedes $y_{i}$ in $\alpha$ and $h_{m}\left(y_{m}\right) \subset$ $h_{i}(q)$ (since $y_{i}$ is the first point $\alpha$ in $\alpha$ such that $x \in h_{i}(a)$ and $h_{i}(a)$. $[S-R] \neq 0$, and $h_{m}\left(y_{m}\right) \subset h_{i}(q)$ would imply that $\left.h_{i}(q) \cdot[S-R] \neq 0\right)$; hence there is no point $q$ such that $q$ precedes $y_{i}$ in $\alpha$ and $y_{m} \in h_{i}(q)$ and $h_{i}(q) \cdot\left[S-g_{r_{\left(y_{m}\right)}[m-1]+1}\left(y_{m}\right)\right] \not \equiv 0$ (otherwise $h_{i}(q)$ would contain $h_{m}\left(y_{m}\right)$ by definition of $\left.h_{m}\left(y_{m}\right)\right)$. Therefore $\overline{h_{m}\left(y_{m}\right)} \subset h_{i}\left(y_{i}\right)$ since $y_{i} \neq y_{m}$.

Hence by Condition $\mathrm{B}^{\prime}$, there is a natural number $N$ such that if $m>N$, then $h_{m}\left(y_{m}\right) \subset R$ contrary to the supposition that for each $i$, $h_{i}\left(y_{i}\right) \cdot[S-R] \neq 0$.

Using the same argument down to the last sentence, which is the 
first place that Condition $\mathrm{B}^{\prime}$ is used, Theorem 3.7 below also follows.

THEOREM 3.7. Suppose that $S$ is a regular semi-metric space. Then there is a basis $H=\left\{h_{n}(x): x \in S, n=1,2, \cdots\right\}$ with the property that for each $p \in S$ and for each closed and compact subset $M$ of $S-p$ there is a natural number $N$ such that if $m>N$ and $p \in h_{m}(x)$ then $h_{m}(x) \cdot M=0$.

4. Completeness axioms. Another way to generalize Moore's Arc 'Theorem is to weaken the completeness used. Three successively weaker completeness axioms $\left(1,1^{\prime}\right.$, and $\left.1^{\prime \prime}\right)$ are given below. In a Moore space: Completeness Axiom 1 is equivalent to Moore's completeness, which is known to be weaker than strong completeness [11, Example 3.3]; and Completeness Axioms 1' and 1" are both equivalent to the completeness in Mrs. Rudin's Axiom 1" [16, p. 320], and hence weaker than Moore's Completeness [16, p. 324]. In semi-metric spaces Completeness Axiom $1^{\prime}$ is stronger than $1^{\prime \prime}$ (all examples in $\S 11$ satisfy Axiom $1^{\prime \prime}$ but not $1^{\prime}$; also see Corollary 4.3).

A Cauchy (or weakly) complete semi-metric space satisfies Completeness Axiom 1". In a metric space all of the completenesses mentioned are equivalent [15].

The theorems listed below (for proofs see [4, pp. 35-43]) give the relationships between the three completeness axioms and the topological properties defined in $\S$ III. Aside from finding that completeness Axioms $1^{\prime}$ and $1^{\prime \prime}$, which are used in separate arc theorems, are more general than Moore's completeness, the main results obtained in this section are (1) that a strongly complete regular semi-metric space is a Moore space (2) that Cauchy (or weak) completeness is weaker than Moore's completeness and (3) a generalization of Theorem 120 in [14] (also Theorem 6 of [16]).

Suppose that $S$ is a $T_{1}$-space and $G=\left\{g_{n}(x): x \in S, n=1,2,3 \cdots\right\}$ is a basis for $S$ which satisfies Condition $\mathrm{A}^{\prime}$. Consider the following completeness axioms for $G$.

Completeness Axiom 1. If $M$ is a nonincreasing sequence of closed sets such that, for each $n$, there is a point $x_{n}$ of $S$ such that $M_{n} \subset$ $g_{n}\left(x_{n}\right)$, then $\prod_{n=1}^{\infty} M_{n} \neq 0$.

Completeness Axiom 1'. If $M$ is a nonincreasing sequence of closed sets and $x$ a point sequence in $S$ such that, for each $n, M_{n} \subset g_{n}\left(x_{n}\right)$ and there is a natural number $k$ such that $\overline{g_{n+k}\left(x_{n+k}\right)} \subset g_{n}\left(x_{n}\right)$, than $\prod_{n=1}^{\infty} M_{n} \neq 0$.

Completeness Axiom 1". If $x$ is a point sequence such that, for 
each $n$, there is a $k$ such that $\overline{g_{n+k}\left(x_{n+k}\right)} \subset g_{n}\left(x_{n}\right)$, then $\prod_{n=1}^{\infty} g_{n}\left(x_{n}\right) \neq 0$.

Theorem 4.1 shows in particular that Axioms 1' and 1" are equivalent in a developable $T_{1}$-space.

THEOREM 4.1. Suppose that $S$ is a $T_{1}$-space with a basis $G$ that satisfies Conditions $A^{\prime}$ and $B^{\prime}$. A necessary and sufficient condition that $G$ satisfy Completeness Axiom $1^{\prime}$ is that $G$ satisfy Completeness Axiom 1".

An immediate corollary to Theorems 4.2 and 3.6 is that a strongly complete regular semi-metric space is a Moore space.

THEOREM 4.2. Suppose that the $T_{2}$-space $S$ has a basis $G$ which satisfies Condition $A^{\prime} . A$ necessary and sufficient condition that $G$ satisfy Condition $B^{\prime}$ and Completeness Axiom $1^{\prime \prime}$ is that $G$ satisfy Completeness Axiom 1'.

CoRollary 4.3. If the regular $T_{1}$-space $S$ has a basis $G$ which satisfies Condition $A$ and Completeness Axiom 1', then $S$ has a basis which satisfies Conditions $A$ and $B$. Hence every strongly complete. regular semi-metric space is a complete Moore space.

The following theorem shows that the space having a basis with some of the completeness properties and another basis with some of the topological properties has a basis with the combined properties.

THEOREM 4.4. If the $T_{1}$-space $S$ has a basis $G$ which satisfies Condition $A^{\prime}$ and one of the Completeness Axioms 1, 1', and 1", and if $S$ has a basis $H$ which satisfies some combination of Conditions $A^{\prime}$, $A, B^{\prime}$, and $B$, then $S$ has a basis $K$ which satisfies the Completeness Axiom that $G$ satisfies and the combination of Conditions $A^{\prime}, A, B^{\prime}$, and $B$ that $H$ satisfies.

The next theorem is a generalization of a portion of a theorem due to Moore [14, p. 83, Theorem 120]. (The other part of the theorem also holds in any of the same spaces). Essentially the same proof may be used (see [4, p. 39]).

THEOREM 4.5. Suppose that the $T_{1}$-space $S$ has a basis $G$ which satisfies Condition $A^{\prime}$ (or $A^{\prime}$ and $A, B^{\prime}$, or $B$ ) and one of the Completeness Axioms 1, 1', and 1". If $M$ is an inner limiting subset (i.e. a $G_{\delta}$ set) of $S$, then there is a basis $H$ for $M$ such that $H$ satisfies the same combination of Conditions $A^{\prime}, A, B^{\prime}$, and $B$ and the Completeness Axiom that $G$ satisfies. 
The completeness defined in Definition 4.6 is clearly weaker in general than Axiom 1'; however, Theorem 4.7 shows that replacing the latter by the former in an arc theorem would not be a real generalization.

Definition 4.6. A basis $G$ for a $T_{1}$-space $S$ which satisfies Condition $\mathrm{A}^{\prime}$ is said to satisfy Completeness Axiom 1' peripherally provided that, if $R \in G, M$ is a nonincreasing sequence of closed subsets of $B(R)$, the boundary of $R$, and $x$ is a point sequence in $S$ such that, for each $n, M_{n} \subset g_{n}\left(x_{n}\right)$ and there is a $k$ such that $\overline{g_{n+k}\left(x_{n+k}\right)} \subset g_{n}\left(x_{n}\right)$, then $\prod_{n=1}^{\infty} M_{n} \neq 0$.

THEOREM 4.7. If $G$ is a basis for a $T_{2}$-space $S$, if each member of $G$ is connected, and if $G$ satisfies Condition $A^{\prime}$ and satisfies Completeness Axiom $1^{\prime}$ peripherally, then $G$ satisfies Condition $B^{\prime}$.

For a proof see [4, pp. 42-43].

5. Metrization theorems. The metrization theorems below serve not only to eliminate certain hypotheses from consideration for generalizing the Arc Theorem, but also to show that the spaces described in $\S I I$, which are not even developable (and in some of which the Arc Theorem does not hold) are nonetheless very close to being metrizable.

Theorem 5.1 generalizes a well-known theorem which is included in Theorem 10 [21]. It can also be shown that every semi-metric space contains a dense metric subspace (but not necessarily one which is an inner limiting subset).

The proof of the following lemma is exactly analogous to the proof of Theorem 15 in $[14$, p. 11].

Lemma. Suppose that $S$ is a regular $T_{1}$-space with a basis $G$ that satisfies Condition $A^{\prime}$ and the Completeness Axiom 1". No closed subset $M$ of $S$ is the sum of countably many closed sets each of which is contained in the boundary of its complement (in $M$ ).

THEOREM 5.1. If the regular $T_{1}$-space $S$ has a basis $G$ that satisfies Condition $A^{\prime}$ and the Completeness Axiom 1", then $S$ contains a dense inner limiting subset $K$ which (with the relative topology) is metrizable and complete.

Proof. Let $H_{1}$ be a maximal collection of mutually exclusive regions each of which belongs to $\left\{g_{i}(x): x \in S, i \geqq i\right\}$. For each $n>1$, let $H_{n}$ be a maximal collection of mutually exclusive regions each of which belongs to $\left\{g_{i}(x): x \in S, i \geqq n\right.$, and there is a region $h$ in $H_{n-1}$ such that $\left.g_{i}(x) \subset h\right\}$. Let $K=\prod_{n=1}^{\infty}\left[H_{n}^{*}\right]$. Since by the above lemma, if $R$ is a 
region in $G, \bar{R}$ is not the sum of countably many closed sets each contained in the boundary of its complement (in $\bar{R}$ ), so that $\bar{R} \neq$ $\sum_{n=1}^{\infty}\left[\left(S-H_{n}^{*}\right) \cdot \bar{R}\right]$ and $\bar{R} \cdot K \neq 0$, it follows that $K$ is dense in $S$. Clearly $K$ is an inner limiting set and $K$ (with the relative topology) is metrizable since $\left(\sum_{n=1}^{\infty} H_{n}\right) \cdot K$ forms a basis for the relative topology of $K$, and since, for each $n$, the elements of $H_{n}$ are pairwise disjoint and each member of $H_{n+1}$ is a subset of a member of $H_{n}$. That $K$ is complete follows from Theorem 4.5.

Theorem 5.2 shows how close the spaces of Examples 2.2, 2.5, 2.7, and 2.8 are to being metrizable. Note that each of those spaces satisfies the hypotheses of the theorem except for being locally peripherally locally compact instead of locally peripherally compact.

THEOREM 5.2. Suppose that the semi-metric space S has a distance function $d$ such that d-neighborhoods are connected sets and such that, if $p$ is a sequential limit point of the point sequence $x$ and $q$ is a sequential limit point of the point sequence $y$ and $\lim _{i \rightarrow \infty} d\left(x_{i}, y_{i}\right)=0$, then $p=q$. If $S$ is locally peripherally compact, then $S$ is metrizable.

Proof. The theorem will be proved by showing that $S$ satisfies the hypothesis of W. A. Wilson's theorem [20, pp. 361 and 366; also 2, p. 63] that a semi-metric space is metrizable provided that, for every pair $x, y$ of sequences, if $p$ is a sequential limit point of $x$ and $\lim _{i \rightarrow \infty} d\left(x_{i}, y_{i}\right)=0$, then $p$ is a sequential limit point of $y$. For suppose that $p$ is a point of $S$ and $x$ and $y$ are point sequences such that $p$ is a sequential limit point of $x$ and $\lim _{i \rightarrow \infty} d\left(x_{i}, y_{i}\right)=0$, but $p$ is not a sequential limit point of $y$. Then there is a region $R$ with compact boundary, $B(R)$, and a sequence $y_{1}^{\prime}, y_{3}^{\prime}, y_{3}^{\prime}, \cdots$ of $y$ such that $R$ contains $p$ but contains none of the points $y_{1}^{\prime}, y_{2}^{\prime}, y_{3}^{\prime}, \ldots$; thus (noting that it may be assumed, without loss of generality, that, for each $i, x_{i} \in R$ and $\left.d\left(x_{i}, y_{1}^{\prime}\right)<(1 / i)\right)$ each of the connected neighborhoods $U_{1}\left(x_{1}\right), U_{1 / 2}\left(x_{2}\right)$, $U_{1 / 3}\left(x_{3}\right), \cdots$ contains a point of $(S-R)$ and hence contains a point of $B(R)$. Let $z$ be a point sequence such that, for each $n, z_{n} \in U_{1 / n}\left(x_{n}\right) \cdot B(R)$. Since $B(R)$ is compact, there is a point $q$ of $B(R)$ such that $q$ is a sequential limit point of asubsequence of $z$, which leads to a contradiction of the hypothesis of the theorem.

By Theorem 5.3 paracompactness (defined along with pointwise paracompactness in [1. p. 177]) is too restrictive in the presence of a completeness axiom slightly stronger than that possessed by the spaces in $\S I I$.

THEOREM 5.3. Suppose that the regular $T_{1}$-space $S$ has a basis $G$ which satisfies Condition $A$ and Completeness Axiom 1' peripherally and whose elements are connected sets. If $S$ is pointwise paracompact, 
then $S$ is developable; hence, if $S$ is paracompact, then $S$ is metrizable.

For a proof of this theorem see [4, pp. 47-48].

6. An arc theorem. Theorem 6.2 is more general than R. L. Moore's Arc Theorem [14, p. 86], to the extent that the completeness axiom used is known to be less restrictive than that in the hypothesis of Moore's theorem, and the other properties used are at least formally more general than those used by Moore. To adapt the proof of Moore's theorem to Theorem 6.2, however, requires only fairly minor modifications. Theorem 6.4 establishes that certain semi-metric spaces which are not arc-wise connected (including Example 2.2) are nontheless connectable by closed connected sets which closely resemble arcs.

In the following definition, which will be used in the proof of Theorem 6.2, "simple chain" (or "chain") is as defined in [14, p. 56].

Definition 6.1. Suppose that $G=\left\{g_{i}(x): x \in S, i=1,2,3, \cdots\right\}$ is a basis for the $T_{1}$-space $S$, that $A$ and $B$ are two points of $S$, and that, for each $n, C_{n}=\left\{Q[n, 1], Q[n, 2], \cdots, Q\left[n, m_{n}\right]\right\}$ is a simple chain from $A$ to $B$. The sequence $\left\{C_{1}, C_{2}, C_{3}, \cdots\right\}$ has property $P$ with respect to $G$ if

(1) for each $i\{\overline{Q[1+i, j]}\}_{j=1}^{m_{i+1}}$ is a refinement of $\{Q[i, j]\}_{j=1}^{m_{i}}$;

(2) for each $i$ and each $j$ such that $1<j \leqq m_{i}$ there is a $K$ such that $\left(\sum_{P<K} Q[i+1, P]\right) \cdot Q[i, j]=0$, and such that $\left(\sum_{P>K} \overline{Q[i+1, P)} \subset\right.$ $\left(\sum_{q=j}^{m_{i}} Q[i, q]\right)$ and $\overline{Q[i+1, k+1]} \subset Q[i, j-1] \cdot Q[i, j]$; and

(3) there is a collection of natural numbers $\{k[i, j]: j \leqq m, i=$ $1,2,3, \cdots\}$ and a collection of points $\left\{\begin{array}{c}x_{i j} \\ 1 \leqq j \leqq m_{i}\end{array}\right\}^{\infty}$ i=1 such that, for each $i$ and each $j \leqq m_{i}$,

(a) $k[i, j] \geqq i$

(b) $Q[i, j]=g_{k(i, j)}\left(x_{i j}\right)$ and

(c) if $x_{i+1, j} \in Q[i, t]$, then $\overline{Q[i+1, j]} \subset Q[i, t]$.

THeOREM 6.2. If the connected regular $T_{1}$-space $S$ has a basis $G$ each of whose elements is connected and which satisfies Condition $A^{\prime}$ and Completeness Axiom 1', then $S$ is arc-wise connected.

Proof. Let $a$ and $b$ be two points of $S$. Moore's proof (in particular Theorem $77[14$, p. 56]) may be applied with slight alterations to the sequence $G$ of open coverings of $S$, such that, for each $n, G_{n}=\left\{g_{k}(x)\right.$ : $x \in S$ and $k \geqq n\}$ to obtain a sequence, $\left\{C_{i}\right\}_{i=1}^{\infty}=\{Q[i, 1], Q[i, 2], \cdots$, $\left.Q\left[i, m_{i}\right]\right\}_{i=1}^{\infty}$, of chains from $a$ to $b$ which has property $P$ with respect to $G$.

Let $M=\prod_{i=1}^{\infty} C_{i}^{*}=\prod_{i=1}^{\infty}\left[\sum_{j=1}^{m_{i}} \overline{Q[i, j]}\right.$. 
Clearly $M$ is closed.

Also $M$ is compact. For suppose that an infinite subset $K$ of $M$ has no limit point. Then there exists a sequence $\left\{H_{n}\right\}_{n=1}^{\infty}$ of collections of pointsets such that, for each $n$ :

(i) each point set in $H_{n}$ is a link of the chain $C_{n}$,

(ii) each set in $H_{n}$ contains an infinite subset of $K$,

(iii) $H_{n+1}$ is a refinement of $H_{n}$, and

(iv) (by the above property $3 c$ of the sequence $C$ ) if $x \in H_{n+1}$, $y \in H_{n}$, and $x \subset y$, then $\bar{x} \subset y$. By theorem $78[14$, p. 56] there is a sequence $h$ such that, for each $n, h_{n} \in H_{n}$ and $h_{n+1} \subset h_{n}$, so that, by the above property (iv) of the sequence $H, \overline{h_{n+1}} \subset h_{n}$. For each $i, h_{i}$ is a link of the chain $C_{i}$, so that, by property $3 b$ (above) of the sequence $C$, there is an increasing natural number sequence $d$ and a point sequence $u$ such that, for each $i, h_{i}=g_{d(i)}\left(u_{i}\right)$, hence, for each $i$, the closed set $\left(h_{i} \cdot K\right)$ is contained in $g_{d(i)}\left(u_{i}\right),\left(h_{i} \cdot K\right) \supset\left(h_{i+1} \cdot K\right)$, and $\overline{g_{d(i+1)}\left(u_{i+1}\right)} \subset g_{d(i)}\left(u_{i}\right)$. Therefore, by Completeness Axiom 1', there is a point $p$ such that $p \in \prod_{i=1}^{\infty}\left[K \cdot h_{i}\right]$; hence, $p \in K$, and for each $i, p \in g_{d(i)}\left(u_{i}\right)$, so that (by Condition $\left.A^{\prime}\right) p$ is a sequential limit point of a subsequence $\left\{u_{t(i)}\right\}_{i=1}^{\infty}$ of the sequence $u$. Consider then the nonincreasing sequence of closed sets, $\left\{(K-p) \cdot h_{t(i)}\right\}_{i=1}^{\infty}$, which likewise has the property that, for each $i$, $\left[(K-p) \cdot h_{t(i)}\right] \subset g_{d[t(i)]}\left(u_{t(i)}\right)$. Again, there is a point $q$ such that $q \in(K-p)$ and such that $q$ is a sequential limit point of $\left\{u_{t(i)}\right\}_{i=1}^{\infty}$ and $p \neq q$. Thus the assumption that $M$ is not compact leads to a contradiction.

That $M$ is connected follows as in Moore's proof with slight alterations (or see [4, pp. 53-54]); and that each point of $M-(a+b)$ is a separating point of $M$ and that $a$ and $b$ are nonseparating points also follows as in Moore's proof.

CoRollary. The connected regular $T_{1}$-space $S$ is arc-wise connected if $S$ satisfies any one of the following conditions:

(a) $S$ has a basis $G$ which satisfies Conditions $\mathrm{A}^{\prime}$ and $\mathrm{B}^{\prime}$ and Completeness Axiom 1" and each of whose elements is connected;

(b) $S$ has a basis $G$ which satisfies Condition $\mathrm{A}^{\prime}$ and satisfies Completeness Axiom $1^{\prime}$ peripherally and each of whose elements is connected;

(c) $S$ is locally connected and satisfies Mary Ellen Estill Rudin's Axiom 1" [16].

(d) $S$ is a locally connected strongly complete semi-metric space.

"Strong chainability," defined below, is a rather restricted special case of chainability but is useful for showing that certain spaces which are not arc-wise connected are connectable by sets which closely resemble arcs. Roughly speaking a space is strongly chainable with respect to 
a basis $G$ provided that, for every pair $a, b$ of points of $S$, there is a sequence $C$ of chains from $a$ to $b$ such that

(1) $C$ has Property $P$ with respect to $b$

(2) the "centers" of $C(n)$ are also "centers" of $C(n+1)$ and

(3) the intersection of two adjacent links of $C(n)$ with centers $p$ and $q$ contains a point $y$ such that the subchain from $p$ to $q$ of $C(n+1)$ is contained in $g_{n}(y)$ and $y$ is a "center" of $C(n+1)$. Theorem 6.2 then establishes that the space of Example 2.2 is connectable by sets having all the properties of an arc but compactness.

Definition 6.3. Suppose that the regular $T_{1}$-space $S$ has a basis, $G=\left\{g_{n}(x): x \in S, n=1,2, \cdots\right\}$, which satisfies Condition A. $S$ is strongly chainable with respect to $G$ provided that, if $a$ and $b$ are two points of $S$, there is a sequence, $C=\left\{Q[i, 1], Q[i, 2], \cdots, Q\left[i, m_{i}\right]\right\}_{i=1}^{\infty}$ of chains from $a$ to $b$ such that:

(1) $C$ has property $P$ with respect to $G$.

(2) There is a collection, $\left\{t(i, j): j \leqq m_{i}, i=1,2, \cdots\right\}$ of natural numbers such that, for each $i$ and each $j \leqq m_{i}, x_{i j}=x_{i+1, t(i, j)}$ : and

(3) there is a collection $\left\{y_{i j}: j<m_{i}, i=1,2, \cdots\right\}$ of points such that, for each $i$ and each $j<m_{i}$, (a) $y_{i j} \in Q[i, j] \cdot Q[i, j+1]$ and $\sum_{r=t(i, j)}^{t(i, j+1)} Q[i+1, r] \subset g_{i}\left(y_{i j}\right)$ and (b) there is a natural number $r$ such that $y_{i j}=x_{i+1, r}$. For each $i$ and each $j \leqq m_{i}$, the point $x_{i j}$ (from Definition 6.1 part (3)) will be referred to as the center of the link $Q[i, j]$ of $C_{i}$.

Note that the space of Example 2.2 (§ II), which is not arc-wise connected, is strongly chainable with respect to the basis consisting of all $1 / n$ neighborhoods (for $n=1,2, \cdots$ ).

THEOREM 6.4. Suppose that $S$ is a connected regular $T_{1}$-space which has a basis, $G=\left\{g_{n}(x): x \in S, n=1,2, \cdots\right\}$, that satisfies Condition $A$ and Completeness Axiom 1" and each of whose elements is connected. If $S$ is strongly chainable with respect to $G$, then, for each pair of points $a$ and $b$, there is a continuum $M$ containing $a$ and $b$ such that $a$ and $b$ are the only non-separating points of $M$.

Proof. Let $a$ and $b$ be two points of $S$; let $C=\{Q[i, 1], Q[i, 2], \cdots$, $\left.Q\left[i, m_{i}\right]\right\}_{i=1}^{\infty}$ be a sequence of chains from $a$ to $b$ satisfying Definition 6.3 and, for each $i$ and each $j \leqq m_{i}$, let $x_{i j}$ be the center of $Q[i, j]$. Denote by $L$ the set of all centers $\left\{\left\{x_{i j}: j \leqq m_{i}, i=1,2, \cdots\right\}\right.$; and let $M=\prod_{i=1}^{\infty}\left[C_{i}\left[^{*}\right.\right.$. Clearly $(a+b) \subset M, M$ is closed, and $a$ and $b$ are the only nonseparating points of $M$.

Furthermore, $M$ is connected. For suppose that $M=H+K$, $\bar{H} \cdot K=H \cdot K=0$ (where $a \in H$ ). Because $M$ is closed, each of $H$ and $K$ is a closed set. Also, because $\bar{L}=M, L \cdot H \neq 0$ and $L \cdot K \neq 0$. It 
will now be shown that there is a natural number $n$, a natural number sequence $u$, and point sequences $p$ and $q$ such that, for each $i \geqq n$, $u(i) \leqq m_{i}$ and

(1) $\overline{Q[i+1, u(i+1)]} \subset Q[i, u(i)]$ and

(2) $p_{i} \in H, q_{i} \in K$, and $\overline{Q[i+1, u(i+1)]} \subset g_{i}\left(p_{i}\right) \cdot g_{i}\left(q_{i}\right)$. Since $a \in H$, since $L \cdot H \neq 0$, and since $L \cdot K \neq 0$, there is a natural number $n$ and a natural number $j<m_{n}$ such that $x_{n j} \in H$ and $x_{n, j+1} \in K$. If $y_{n j} \in K$, let $u(n)=j$; if $y_{n j} \in H$, let $u(n)=j+1$. In either case there is a natural number $r$ such that $x_{n+1, r} \in H, x_{n+1, r+1} \in K$, and $\overline{[Q[n+1, r]}+\overline{Q[n+1}$, $\overline{r+1]]} \subset Q[n, u(n)] \cdot g_{n}\left(y_{n j}\right)$; again, if $y_{n+1, r} \in K$, let $u(n+1)=r$, and if $y_{n+1, r} \in H$, let $u(n+1)=r+1$; and the process may be continued to define sequences $u, p$, and $q$ which have the stated properties. Then, by Completeness Axiom $1^{\prime \prime}$ there is a point $z$ such that $z \in \prod_{n=1}^{\infty} \overline{Q[n+1}$, $\overline{u(n+1)]}$ and $z$ is a sequential limit point of the sequence $p$ in $H$ and of the sequence $q$ in $K$; hence $z \in H \cdot K$ contrary to the assumption that $H \cdot K=0$.

7. Summary and questions. Theorem 2.3 establishes that Moore's Arc Theorem cannot be generalized directly to Cauchy complete regular semi-metric spaces, while Theorem 6.2 shows that it can be generalized to a class of semi-metric spaces somewhat more general than complete Moore spaces-in particular, the completeness axiom used is known to be weaker than that of Moore's Axiom 1. The examples in $\S$ II and the theorems establishing certain sufficient conditions for a semi-metric space to be developable or even metrizable given in $\S$ III, IV and V show rather clearly the limited nature of the progress that can be made towards extending the arc Theorem to semi-metric spaces. For example, Theorems 3.6 and 4.2 establish that every strongly complete regular semi-metric space is a complete Moore space.

The following questions then are suggested:

(1) Can Moore's Arc Theorem be generalized in another direction, such as to complete uniform spaces?

(2) Since the class of strongly complete regular semi-metric spaces properly includes the class of all complete Moore spaces and is properly included in the class of all complete metric spaces, what is a sufficient condition for a complete Moore space-or a weakly complete semi-metric space - to be strongly complete, and what is a sufficient condition for a strongly complete regular semi-metric space to be metrizable?

(3) Is there any reasonable necessary and sufficient condition for a connected and locally connected complete regular semi-metric space to be arc-wise connected? 
1. R. H. Bing, Metrization of topological spaces, Canadian J. of Math., 3 (1951), 175-186.

2. Morton Brown, Semi-metric spaces Summer Institute on Set Theoretic Topology, Madison, Wisconsin, Amer. Math. Soc., (1955), 62-64.

3. L. W. Cohen, Uniformity in topological spaces, Lectures in Topology ed. by Wilder and Ayres, University of Michigan Press, (1941), 255-265.

4. R. W. Heath, Arc-wise connectedness in semi-metric spaces, doctoral dissertation, University of North Carolina, 1959.

5. — A regular semi-metric space for which there is no semi-metric under which all spheres are open, Proc. Amer. Math. Soc., 12 (1961), 810-811.

6. F. B. Jones, Connected and disconnected plane sets and the functional equations $f(x)+$ $f(y)=f(x+y)$, Bull. Amer. Math. Soc., 48 (1942), 115-120.

7. - Introductory remarks on semi-metric spaces, Summer Institute on Set Theoretic Topology, Madison, Wisconsin, Amer. Math. Soc. (1955), 58.

8. — R. L. Moore's Axiom 1 and metrization, Proc. Amer. Math. Soc., 9 (1958), 487.

9. J. L. Kelley, General Topology, Princeton: D. Van Nostrand Company, 1955.

10. C. Kuratowski, Topologie II, Warsaw, (1950), 184.

11. L. F. McAuley, A Relation between perfect separability, Completeness, and normality in semi-metric spaces, Pacific J. Math., 6 (1956), 315-326.

12. - On semi-metric spaces, Summer Institute on Set Theoretic Topology, Madison, Wisconsin, Amer. Math. Soc., (1955), 58-62.

13. R. L. Moore, Abstract sets and foundations of analysis situs, Bull, Amer. Math. Soc., 33 (1927), 141.

14. - Foundations of point set theory, Amer. Math. Soc. Coll. Publ., 13, New York: Amer. Math. Soc., 1932.

15. J. H. Roberts, A property related to completeness, Bull. Amer. Math. Soc., 38 (1932), 835-838.

16. Mary Ellen Estill Rudin, Concerning abstract spaces, Duke Math. J., 17 (1950), 317-327. 17. A. H. Stone, Paracompactness and product spaces, Bull. Amer. Math., Soc., 54 (1948), 977-982.

18. C. W. Vickery, Moore spaces and metric spaces, Bull. Amer. Math. Soc., 46 (1940), 560-564.

19. G. T. Whyburn, Analytic topology, Amer. Math. Soc. Coll. Publ., 28, New York, 1942.

20. W. A. Wilson, On semi-metric spaces, Amer. J. Math., 53 (1931), 361-373.

21. J. N. Younglove, Concerning dense metric subspaces of certain nonmetric spaces, Fundamenta Mathematicae, 48 (1959), 15-25.

The UNIVERsity OF NORTh CAROLINA AND

THE UNIVERSITY OF GEORGIA 



\section{PACIFIC JOURNAL OF MATHEMATICS}

\section{EDITORS}

\author{
Ralph S. Phillips \\ Stanford University \\ Stanford, California \\ M. G. Arsove \\ University of Washington \\ Seattle 5 , Washington
}

\author{
A. L. Whiteman \\ University of Southern California \\ Los Angeles 7, California \\ Lowell J. Paige \\ University of California \\ Los Angeles 24, California
}
E. F. BECKENBACH
D. DERRY
ASSOCIATE EDITORS
T. M. CHERRY
M. OHTSUKA
H. L. ROYDEN
E. G. STRAUS
E. SPANIER
F. WOLF

\section{SUPPORTING INSTITUTIONS}

\author{
UNIVERSITY OF BRITISH COLUMBIA \\ CALIFORNIA INSTITUTE OF TECHNOLOGY \\ UNIVERSITY OF CALIFORNIA \\ MONTANA STATE UNIVERSITY \\ UNIVERSITY OF NEVADA \\ NEW MEXICO STATE UNIVERSITY \\ OREGON STATE UNIVERSITY \\ UNIVERSITY OF OREGON \\ OSAKA UNIVERSITY \\ UNIVERSITY OF SOUTHERN CALIFORNIA
}

\author{
STANFORD UNIVERSITY \\ UNIVERSITY OF TOKYO \\ UNIVERSITY OF UTAH \\ WASHINGTON STATE UNIVERSITY \\ UNIVERSITY OF WASHINGTON \\ AMERICAN MATHEMATICAL SOCIETY \\ CALIFORNIA RESEARCH CORPORATION \\ SPACE TECHNOLOGY LABORATORIES \\ NAVAL ORDNANCE TEST STATION
}

Mathematical papers intended for publication in the Pacific Journal of Mathematıcs should be typewritten (double spaced), and the author should keep a complete copy. Manuscripts may be sent to any one of the four editors. All other communications to the editors should be addressed to the managing editor, L. J. Paige at the University of California, Los Angeles 24, California.

50 reprints per author of each article are furnished free of charge; additional copies may be obtained at cost in multiples of 50 .

The Pacific Journal of Mathematics is published quarterly, in March, June, September, and December. Effective with Volume 13 the price per volume (4 numbers) is $\$ 18.00$; single issues, $\$ 5.00$. Special price for current issues to individual faculty members of supporting institutions and to individual members of the American Mathematical Society: $\$ 8.00$ per volume; single issues $\$ 2.50$. Back numbers are available.

Subscriptions, orders for back numbers, and changes of address should be sent to Pacific Journal of Mathematics, 103 Highland Boulevard, Berkeley 8, California.

Printed at Kokusai Bunken Insatsusha (International Academic Printing Co., Ltd.), No. 6, 2-chome, Fujimi-cho, Chiyoda-ku, Tokyo, Japan.

PUBLISHED BY PACIFIC JOURNAL OF MATHEMATICS, A NON-PROFIT CORPORATION

The Supporting Institutions listed above contribute to the cost of publication of this Journal, but they are not owners or publishers and have no responsibility for its content or policies. 


\section{Pacific Journal of Mathematics}

\section{Vol. 12 , No. 4 \\ April, 1962}

Tsuyoshi Andô, On fundamental properties of a Banach space with a cone ..... . 1163

Sterling K. Berberian, A note on hyponormal operators ................ 1171

Errett Albert Bishop, Analytic functions with values in a Frechet space . . . . . . . 1177

(Sherman) Elwood Bohn, Equicontinuity of solutions of a quasi-linear equation ............................................ 1193

Andrew Michael Bruckner and E. Ostrow, Some function classes related to the class of convex functions . . . . . . . . . . . . . . . . . . . . . . . . 1203

J. H. Curtiss, Limits and bounds for divided differences on a Jordan curve in the complex domain . . ................................. 1217

P. H. Doyle, III and John Gilbert Hocking, Dimensional invertibility . . . . . . . . 1235

David G. Feingold and Richard Steven Varga, Block diagonally dominant matrices and generalizations of the Gerschgorin circle theorem ................. 1241

Leonard Dubois Fountain and Lloyd Kenneth Jackson, A generalized solution of the boundary value problem for $y^{\prime \prime}=f\left(x, y, y^{\prime}\right) \ldots \ldots \ldots \ldots \ldots \ldots \ldots \ldots \ldots$

Robert William Gilmer, Jr., Rings in which semi-primary ideals are primary. . . . . 1273

Ruth Goodman, K-polar polynomials .......................... 1277

Israel Halperin and Maria Wonenburger, On the additivity of lattice completeness ........................................... 1289

Robert Winship Heath, Arc-wise connectedness in semi-metric spaces . . . . . . . . 1301

Isidore Heller and Alan Jerome Hoffman, On unimodular matrices . . . . . . . . . . . 1321

Robert G. Heyneman, Duality in general ergodic theory . . . . . . . . . . . . . . . 1329

Charles Ray Hobby, Abelian subgroups of p-groups . . . . . . . . . . . . . . . 1343

Kenneth Myron Hoffman and Hugo Rossi, The minimum boundary for an analytic

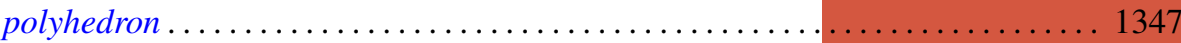

Adam Koranyi, The Bergman kernel function for tubes over convex cones ........ 1355

Pesi Rustom Masani and Jack Max Robertson, The time-domain analysis of a continuous parameter weakly stationary stochastic proces.

William Schumacher Massey, Non-existence of almost-complex structures on

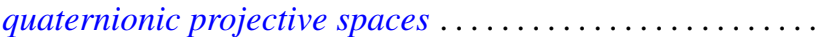

Deane Montgomery and Chung-Tao Yang, A theorem on the action of $\mathrm{SO}(3) \ldots . .1385$

Ronald John Nunke, A note on Abelian group extensions . . . . . . . . . . . . . 1401

Carl Mark Pearcy, A complete set of unitary invariants for operators generating finite $W^{*}$-algebras of type $I$

Edward C. Posner, Integral closure of rings of solutions of linear differential equations.

Duane Sather, Asymptotics. III. Stationary phase for two parameters with an application to Bessel functions.

J. Śladkowska, Bounds of analytic functions of two complex variables in domains

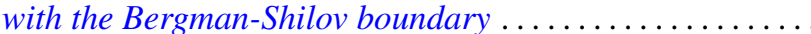

Joseph Gail Stampfli, Hyponormal operators .

George Gustave Weill, Some extremal properties of linear combinations of kernels

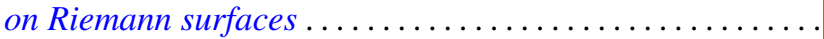

